\title{
Reconstruction of a Total Soft Palatal Defect Using a Folded Radial Forearm Free Flap and Palmaris Longus Tendon Sling
}

\author{
Myung Chul Lee, Dong Won Lee, Dong Kyun Rah, Won Jai Lee \\ Department of Plastic and Reconstructive Surgery, Yonsei University Severance Medical Center, Yonsei University College of Medicine, \\ Seoul, Korea
}

Background The soft palate functions as a valve and helps generate the oral pressure required for normal speech resonance. Speech problems and nasal regurgitation can result from a soft palatal defect. Reduction of the size of the velopharyngeal orifice is required to compensate for the lack of mobility in a reconstructed soft palate. We suggest a large volume folded free flap for reduction of the caliber and a palmaris longus tendon sling for suspension of the reconstructed palate.

Methods Six patients had total soft palate resection for tonsillar cancer and reconstruction with a large volume folded radial forearm free flap combined with a palmaris longus sling. A single surgeon and speech therapist examined the patients with three standardized speech assessment tools: nasometer test, consonant articulation test, and speech acuity test performed for speech evaluation.

Results Mean nasalance score was $76.20 \%$ for sentences with nasal sounds and $43.60 \%$ for sentences with oral sounds. Hypernasality was seen for oral sound sentences. The mean score of the picture consonant articulation test was $84 \%$ (range, 63\% to 100\%). The mean score of the speech acuity test was 5.84 (range, 5 to 6 ). These mean ratings represent a satisfactory level of speech function.

Conclusions The large volume folded free flap with a palmaris longus tendon sling for total soft palate reconstruction resulted in satisfactory prognosis for speech despite moderate hypernasality.

Keywords Palate, soft / Free tissue flaps / Speech
Correspondence: Won Jai Lee Department of Plastic and Reconstructive Surgery, Yonsei University Severance Medical Center, Yonsei University College of Medicine, 50 Yonsei-ro, Seodaemun-gu, Seoul 120-752, Korea

Tel: $+82-2-2228-2210$

Fax: +82-2-361-6947

E-mail: pswjlee@yuhs.ac

This article was presented at the 65th Congress of the Korean Society of Plastic and Reconstructive Surgeons on November 6-8, 2008 in Seoul, Korea.

No potential conflict of interest relevant to this article was reported.

\section{INTRODUCTION}

The reconstruction of a soft palatal defect due to head and neck cancer surgery remains a challenge because of velopharyngeal incompetence resulting from the wide resection. The soft palate combines pharyngeal walls at the velopharyngeal aperture to function as a valve and to generate the oral pressure required for normal speech resonance and bolus propulsion during swallowing [1]. The total soft palatal defect can result in speech problems and nasal regurgitation. Therefore, reconstruction of the soft palate should not only cover the defect, but also provide functional restoration of the levator mechanism to obtain velo-

Copyright $\odot 2012$ The Korean Society of Plastic and Reconstructive Surgeons

This is an Open Access article distributed under the terms of the Creative Commons Attribution Non-Commercial License (http://creativecommons.org/

licenses/by-nc/3.0/) which permits unrestricted non-commercial use, distribution, and reproduction in any medium, provided the original work is properly cited.

www.e-aps.org 
pharyngeal closure.

The palatal local flap, folded free flap [2,3], and free flap combined with pharyngeal flap [4] have been introduced. However, the resulting speech abilities have been shown to be unsatisfactory and variable. Simple coverage of a soft palatal defect is inadequate and reduction of the caliber of the velopharyngeal aperture is required to compensate for the lack of mobility in the reconstructed palate [5]. Previously, we reported the radial forearm tenocutaneous free flap including a palmaris longus tendon sling for moderate-sized defects and proved the efficacy of the procedure as applied to up to half of a soft palate defect [6].

In this study, we propose a large volume folded free flap for the reduction of the caliber and a palmaris longus sling for the suspension of the reconstructed palate to improve the prognosis for speech in the case of a total soft palate defect.

\section{METHODS}

Six patients who had undergone total soft palate resection followed by reconstruction with a large volume folded free flap and palmaris longus tendon sling during the two-year period between 2006 and 2008 were reviewed. They included five males and one female. The patients ranged in age from 41 to 68 years, with a mean age of 56.5 years. All patients had undergone total soft palatal and partial oropharyngeal resections for tonsillar cancer.
Both the oral and nasal sides of the soft palate were designed according to our previously published flap design method [7]. A large volume fasciocutaneous free flap was folded over upon itself to provide a mucosal lining substitute for both the nasal and oral surfaces of the palate. The flap restored the oropharyngeal wall defects in continuity with the palatal reconstruction. After insetting of the flap and suturing of the nasal side, the palmaris longus tendon was placed beneath the fascial surface of the flap. The palmaris longus tendon was sutured to the remaining oropharyngeal musculature, including the levator veli palatini and pharyngeal constrictor muscle, as far posteriorly and superiorly as possible to elevate the posterior margin of the palate effectively (Fig. 1).

To assess nasality and velopharyngeal closure, three evalu-

\begin{tabular}{|lcc|}
\hline Table 1. Nasometer test \\
\hline & Stimulus sentence & $\begin{array}{c}\text { Average } \pm \text { standard } \\
\text { deviation (range) }\end{array}$ \\
\hline Nasal sound sentence & Umma & $60.41 \pm 6.92$ \\
Oral sound sentence & ('Mother' in Korean) & $(47.23-74.25)$ \\
& Appa & $8.78 \pm 4.52$ \\
& ('Father' in Korean) & $(0-17.82)$ \\
& Aaga & $12.66 \pm 7.05$ \\
& ('Baby' in Korean) & $(0-26.76)$ \\
& Yeogi & $19.10 \pm 8.26$ \\
& ('Here' in Korean) & $(2.58-35.62)$ \\
\hline
\end{tabular}

A nasometer test was performed while patients read sentences containing nasal and oral sounds.

\section{Fig. 1. Depiction of surgical procedures}

(A) Oropharyngeal defect including total palatal defect (marked with dot) was noted after oncologic surgery. (B, C) Elevated free flap and palmaris longus tendon (a) placed beneath fascial surface of the flap, "A" point was folded for covering the nasal side. (D) Free flap was inset and palmaris longus $(\mathrm{PL})$ tendon tendon was fastened to the adjacent levator veli palatini and pharyngeal constrictor muscle for suspension.

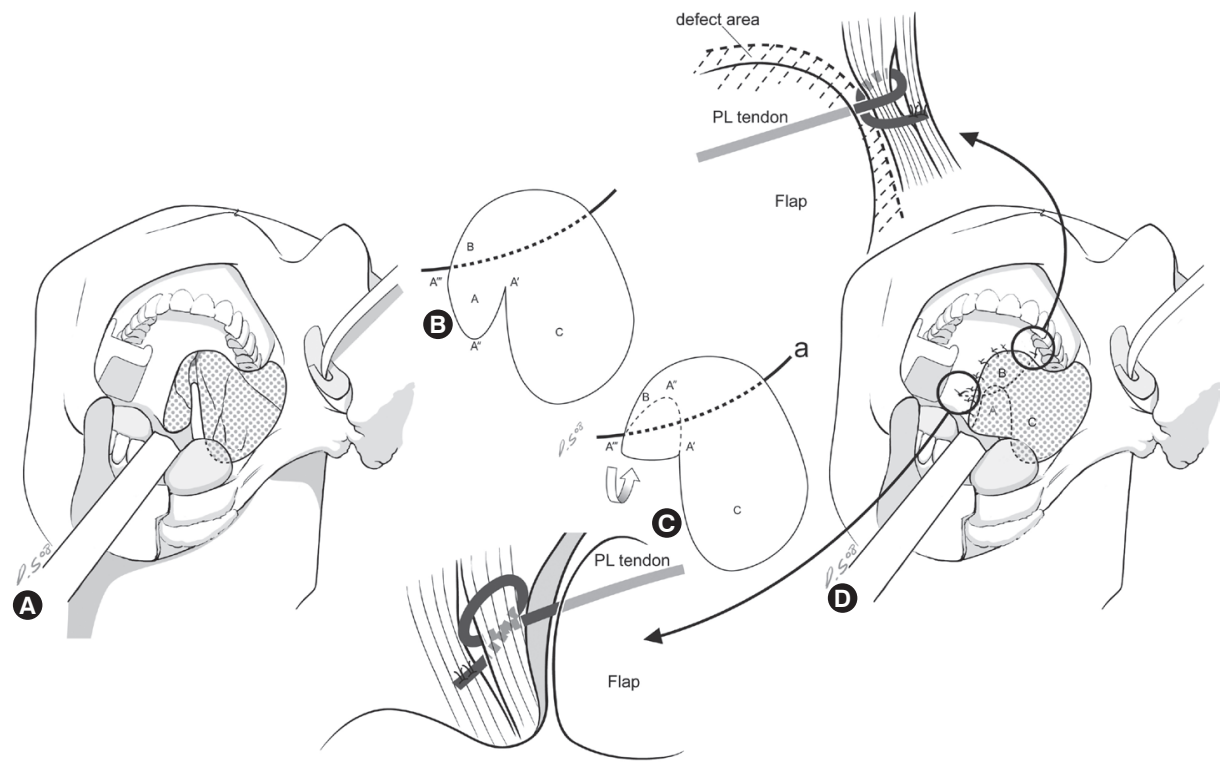


Table 2. Consonant articulation test

\begin{tabular}{|c|c|c|c|c|c|c|c|c|c|c|c|c|c|c|c|c|c|c|c|}
\hline & \multicolumn{3}{|c|}{$\begin{array}{l}\text { Bilabial } \\
\text { plosive }\end{array}$} & \multicolumn{3}{|c|}{$\begin{array}{l}\text { Alveolar } \\
\text { plosive }\end{array}$} & \multicolumn{3}{|c|}{$\begin{array}{l}\text { Velar } \\
\text { plosive }\end{array}$} & \multicolumn{3}{|c|}{$\begin{array}{l}\text { Palatal } \\
\text { plosive }\end{array}$} & \multicolumn{3}{|c|}{ Fricative } & \multicolumn{3}{|c|}{$\begin{array}{l}\text { Nasal } \\
\text { sound }\end{array}$} & \multirow{2}{*}{$\begin{array}{c}\text { Liquid } \\
\text { sound } \\
ᄅ\end{array}$} \\
\hline & $\forall$ & 甘 & II & ᄃ & 匹ᄄ & $E$ & $\neg$ & 77 & $\exists$ & $\pi$ & यु & $\bar{\lambda}$ & $\curlywedge$ & $\mu$ & $\bar{\partial}$ & ㅁ & \llcorner & 0 & \\
\hline & [b] & [p] & {$\left[p^{h}\right]$} & [d] & {$[t]$} & {$\left[\mathrm{t}^{\mathrm{h}}\right]$} & {$[\mathrm{g}]$} & {$[\mathrm{k}]$} & {$\left[k^{h}\right]$} & {$\left[\mathrm{t}_{6}\right]$} & {$\left[\mathrm{d}_{z}\right]$} & {$\left[\mathrm{t}_{6}^{\mathrm{h}}\right]$} & {$[s]$} & [ss] & [h] & {$[\mathrm{m}]$} & [n] & [n] & [I] \\
\hline I & $\mathrm{D}$ & + & + & D & $D$ & + & + & + & D & + & + & + & $D$ & $\mathrm{D}$ & + & + & D & & D \\
\hline$M$ & + & + & + & + & + & + & + & + & D & + & + & + & + & $S$ & + & + & + & & D \\
\hline $\mathrm{F}$ & + & & & + & & & + & & & & & & & & & + & + & + & + \\
\hline
\end{tabular}

The grade for consonant articulation was assigned using a picture consonant articulation test, and the analysis of errors is described in the table. The result of one of the patients (patient 6 in Table 5) is presented on this table as an example.

I, initial consonant; $M$, medial consonant; $F$, final consonant; $D$, distortion; $S$, substitution; 0 , omission; +, correct articulation.

Table 3. Errors in consonant articulation test

\begin{tabular}{|c|c|c|c|c|c|c|c|}
\hline \multicolumn{2}{|c|}{ Articulation method } & \multicolumn{2}{|c|}{ Articulation site } & \multicolumn{2}{|c|}{ The location within a word } & \multicolumn{2}{|c|}{ The type of error } \\
\hline Type & Number & Type & Number & Type & Number & Type & Number \\
\hline Plosive & 5 & Bilabial & 1 & Initial sound & 8 & Distortion & 12 \\
\hline Affricate & 0 & Aveolar & 2 & Middle sound & 3 & Substitution & 1 \\
\hline Fricative & 3 & Palatal & 0 & Final sound & 0 & Omission & 0 \\
\hline Nasal sound & 1 & Velar & 2 & & & & \\
\hline Liquid sound & 2 & Laryngeal & 0 & & & & \\
\hline
\end{tabular}

\section{Table 4. Speech acuity test}

\begin{tabular}{|c|c|}
\hline Score & Standard of reference \\
\hline 7 & No reading difficulty \\
\hline 6 & $<25 \%$ error but can be understood in almost of the reading \\
\hline 5 & $\begin{array}{l}\text { About } 50 \% \text { error but can be understood in almost of the } \\
\text { reading }\end{array}$ \\
\hline 4 & $\begin{array}{l}\text { About } 75 \% \text { error but can be understood if listened to } \\
\text { carefully }\end{array}$ \\
\hline 3 & $>50 \%$ heard in only fragments of words \\
\hline 2 & $<50 \%$ heard in only fragments of words \\
\hline 1 & Cannot be understood, only mumbles \\
\hline \multicolumn{2}{|c|}{$\begin{array}{l}\text { Speech acuity grade on speaking voluntarily chosen sentences and } \\
\text { reading specific sentences. }\end{array}$} \\
\hline
\end{tabular}

ation methods were utilized: i) a nasometer (model 6200-2, Kay Elemetrics Co., Pine Brook, NJ, USA) (nasalance score, $\%)$ (Table 1), ii) the consonant articulation test (Tables 2, 3), and iii) the speech acuity test (Table 4 ) performed by a single speech therapist. Nasalance was measured while patients uttered sentences with specific nasal and oral sounds (Table 1). The consonant articulation precision was assessed through a picture consonant articulation test while patients pronounced various letters (Tables 2, 3). Their speech acuity and intelligibility was evaluated by letting each patient speak voluntarily-chosen sentences and having them read specific sentences. A single surgeon and speech therapist reviewed the phonation of the patients' speech and scored it on a scale of one to seven with a standard-

\section{Fig. 2. Pre- and postoperative figures (patient 3 in table 5)}

(A) A 58-year-old male patient underwent wide excision including supraomohyoid neck dissection on the ipsilateral side for stage III tonsillar cancer. A total soft palatal defect was noted after oncologic surgery. (B) A large volume fasciocutaneous free flap was folded and inset accompanying suspension with a palmaris longus tendon. (C) The reconstructed soft palate maintained its physiologic position postoperatively. (D) Supero-posterior movement of the reconstructed soft palate was observed during phonation. The patient's nasalance score was $83.24 \%$ on nasal sound sentences and $62.54 \%$ on oral sound sentences. The grade of the picture consonant articulation test was $88.37 \%$. The score of the speech acuity test was 6 .
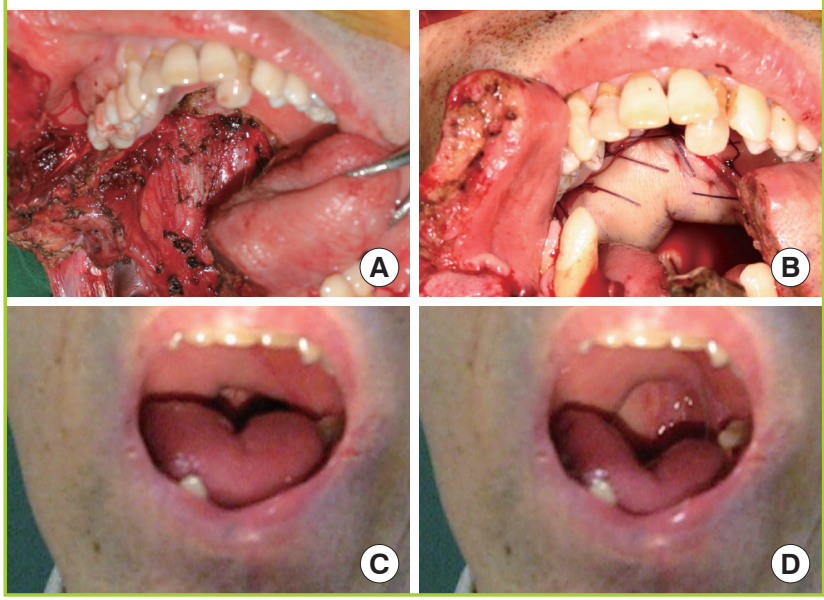
Table 5. Patient characteristics and outcomes

\begin{tabular}{|c|c|c|c|c|c|c|c|c|c|c|c|c|}
\hline \multirow{2}{*}{ Patient } & \multirow{2}{*}{ Sex } & \multirow{2}{*}{ Age } & \multicolumn{2}{|c|}{ Neck dissection } & \multirow{2}{*}{$\begin{array}{c}\text { Pathol- } \\
\text { ogy }\end{array}$} & \multirow{2}{*}{ Stage } & \multirow{2}{*}{$\begin{array}{c}\text { Tx } \\
\text { modality }\end{array}$} & \multirow{2}{*}{$\begin{array}{l}\text { Postop. } \\
\text { RTx } \\
\text { (cGy) }\end{array}$} & \multicolumn{2}{|c|}{ Nasalance score $(\%)$} & \multirow{2}{*}{$\begin{array}{c}\text { Consonant } \\
\text { precision } \\
(\%)\end{array}$} & \multirow{2}{*}{$\begin{array}{l}\text { Speech } \\
\text { acuity }\end{array}$} \\
\hline & & & Ipsilateral & Contralateral & & & & & Nasal & Oral & & \\
\hline 1 & $M$ & 63 & MRND & & SCC & IVa & SR & 4664 & 80.53 & 35.78 & 90 & 6 \\
\hline 2 & $\mathrm{~F}$ & 54 & SOND & & $\mathrm{SCC}$ & IVa & SR & 5400 & $x$ & $x$ & 63 & 6 \\
\hline $3^{\text {a) }}$ & M & 58 & SOND & & SCC & III & SR & 5940 & 83.24 & 62.54 & 88.37 & 6 \\
\hline 4 & M & 68 & Extended RND & MRND & SCC & Ila & SR & 6148 & 69.17 & 53.51 & 90.69 & 6 \\
\hline $5^{\text {a) }}$ & M & 55 & MRND & SOND & SCC & IVa & SR & 5940 & 75.18 & 9.08 & 100 & 6 \\
\hline 6 & M & 41 & Extended RND & MRND & SCC & IVa & SCR & 4680 & 72.87 & 57.12 & 72 & 5 \\
\hline & & & & & & & & & 76.20 & 43.60 & 84 & 5.84 \\
\hline
\end{tabular}

The mean nasalance score was $76.20 \%$ on nasal sound sentences and $43.60 \%$ on oral sound sentences (normal range, $23.75 \%$ to $36.25 \%$ ). The mean score on the picture consonant articulation test was $84 \%$ (range, 63\% to 100\%). The mean score of the speech acuity test was 5.84 (range, 5 to 6). These mean ratings represent a satisfactory level of speech function.

$X$, the patient refused the test; Tx, treatment; MRND, modified radical lymph node dissection; SOND, supraomohyoid lymph node dissection; SCC, squamous cell carcinoma; SR, surgery with radiation therapy; SCR, surgery with chemoradiation therapy.

${ }^{a)}$ Case presented in the article.

\section{Fig. 3. Pre- and postoperative figures (patient 5 in table 5 )}

(A) A 55-year-old male patient underwent wide excision for stage IVa tonsillar cancer. (B) A total soft palatal defect including the partial loss of the lateral and posterior pharyngeal wall was noted after oncologic surgery. (C) A large volume fasciocutaneous free flap was folded and inset accompanying palmaris longus suspension. (D) Elevation of the soft palate was observed during phonation 9 months postoperatively. The patient's nasalance score was $75.18 \%$ on sentences with nasal sounds and $9.08 \%$ on sentences with oral sounds. The grade on the picture consonant articulation test was 100\%. The score on the speech acuity test was 6 .
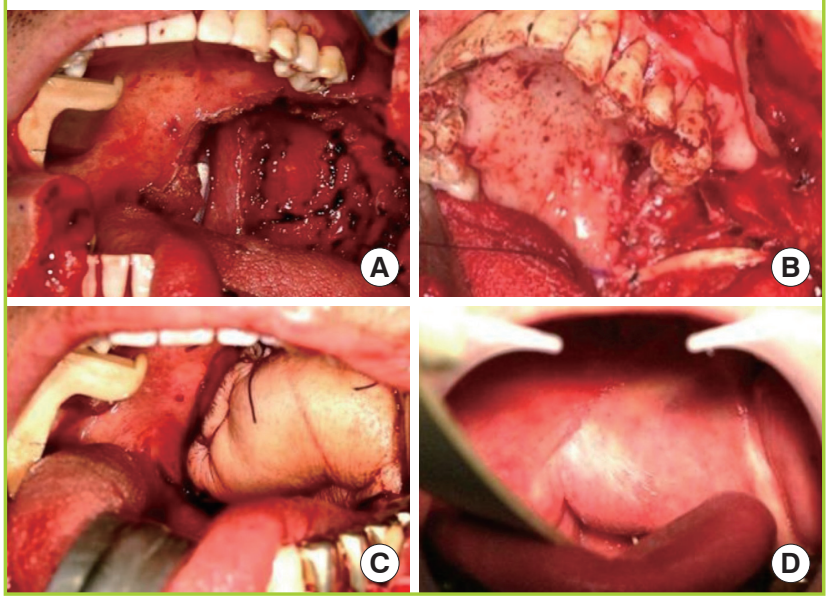

ized speech assessment tool (Table 4). A score over six points was regarded as a normal speech intelligibility.

\section{RESULTS}

All the patients in the series underwent reconstruction with the described method of a radial forearm free flap incorporating suspension with the palmaris longus tendon. There were no wound-related or microsurgical complications in the series. Speech evaluation was performed at a mean 9 months postoperatively.
The mean nasalance score was $76.20 \%$ on nasal sound sentences (normal range, $47.23 \%$ to $74.25 \%$ ) and $43.60 \%$ on oral sound sentences (normal range, $23.75 \%$ to $36.25 \%$ ). Hypernasality was seen on oral sound sentences. The mean score of the consonant articulation test was $84 \%$ (range, $63 \%$ to $100 \%$ ). The mean score of the speech acuity test was 5.84 (range, 5 to 6 ). These mean ratings represent a satisfactory level of speech function (Table 5).

\section{Case 1 (patient 3 in Table 5)}

A 58-year-old male patient underwent wide excision including supraomohyoid neck dissection (SOND) on the ipsilateral side for stage III tonsillar cancer. A total soft palatal defect was noted after oncologic surgery. A large volume fasciocutaneous free flap was folded and inset accompanying suspension with a palmaris longus tendon. Postoperative radiation therapy (5,940 cGy) was performed. Supero-posterior movement of the reconstructed soft palate was observed during phonation postoperatively. The patient's nasalance score was $83.24 \%$ on sentences with nasal sounds and $62.54 \%$ on sentences with oral sounds. The grade on the consonant articulation test was $88.37 \%$. The score of the speech acuity test was 6 , and the resulting speech ability was satisfactory (Table 4, Fig. 2).

\section{Case 2 (patient 5 in Table 5)}

A 55-year-old male patient had a wide excision including a modified radical neck dissection on the ipsilateral side and a SOND on the contralateral side for tonsillar cancer, stage IVa. A total soft palatal defect including the partial loss of the lateral and posterior pharyngeal wall was noted after oncologic surgery. A large volume fasciocutaneous free flap was folded and inset accompanied with a palmaris longus tendon sling. Postoperative radiation therapy $(5,940 \mathrm{cGy})$ was performed. Elevation of 
the reconstructed soft palate was observed during phonation 9 months postoperatively. The patient's nasalance score was $75.18 \%$ on nasal sound sentences and $9.08 \%$ on oral sound sentences. The grade on the consonant articulation test was $100 \%$. The score on the speech acuity test was 6 , and the resulting speech ability was satisfactory (Table 4, Fig. 3).

\section{DISCUSSION}

Head and neck reconstructive surgeons are often confronted with the difficult challenge of the preservation of function, cosmesis, and quality of life when choosing the best operative plan for a patient. This task is especially difficult for cancer of the oropharynx because this site is intimately involved with the complex functions of respiration, deglutition, and speech production [8]. The soft palate is a moving structure that functions as a valve and generates the oral pressure required for normal speech resonance. It is even more difficult to achieve successful functional outcome after total soft palate resection.

Numerous operative techniques have been attempted to improve the speech and swallowing functions after wide resection of oropharyngeal structures including more than half of the soft palate. Brown et al. [4] suggested that the introduction of an additional superiorly based pharyngeal flap results in a functional advantage, especially for three-quarter and total soft palate reconstructions. Rieger et al. [9] analyzed speech data for patients with half or more of the soft palate resected, followed by reconstruction with soft palate insufficiency repair (SPIR). The SPIR procedure includes incising the dermis along the free edge of the folded radial forearm free flap. Small subdermal and submucosal flaps are elevated on the lateral and posterior pharyngeal walls. These flaps are then sutured to the free edge of the folded radial forearm free flap, leaving only a small nasopharyngeal port on one side. Reconstruction with SPIR modification resulted in better speech outcomes. However, these techniques require an uninjured posterior pharyngeal wall during the procedures. McCombe et al. [5] introduced the radial forearm free flap folded over the palmaris longus tendon for the total palatectomy defect. This technique was aimed at restoring the normal palatal anatomy. Maintenance of the reconstructed flap position was attempted by bridging the flap between the lateral pharyngeal wall and the remaining soft palate in the partial palatectomy defects or in the total palatectomy defects, using the palmaris longus tendon as a sling for the posterior margin of the flap. The authors of our institute have published a report of a successful radial forearm palmaris longus tenocutaneous flap for postoncologic soft palate defects, and have observed that incorporating the palmaris longus tendon sling across the remnant levator and the pharyngeal constrictor muscles proved to be beneficial in the dynamic reconstruction of the soft palate in moderate resections of up to half of the soft palate [6].

In advanced staged tumors, greater resection of the soft palate, and the lateral and posterior pharyngeal wall results in an increased risk of velopharyngeal incompetence due to the increased space between the reconstructed soft palate and posterior pharyngeal wall despite the free tissue transfer. Therefore, solely reconstructing the soft palate so that it is anatomically similar is physiologically inadequate and reduction of the cross sectional area in the velopharyngeal space is required to compensate for the lack of mobility in the reconstructed palate. Seikaly et al. [10] reported that larger flaps showed lower nasalance values. We have designed the radial forearm flap to be as large as possible while avoiding obstruction of the airway and oropharyngeal structures. The suspension with the palmaris longus tendon provides a tensile connection between the remnant levator muscle and the pharyngeal muscles, reducing the chances of velopharyngeal incompetence. The suspension procedure also helps to prevent the flaps from being drawn far from the posterior pharyngeal wall as the flap bulk decreases over time and contracts after postoperative radiation therapy.

The nasalance score was $76.20 \%$ for sentences with nasal sounds and $43.60 \%$ for those with oral sounds. Hypernasality was seen in the oral sound sentences. The consonant articulation test and speech acuity test represented clearly good functional results. Relatively poor scores and hypernasality for the oral sound sentences seems to be due to incomplete closure of the velopharyngeal orifice while pronouncing the oral sound. A large quantity of oropharyngeal muscle including the soft palate, and lateral and posterior pharyngeal wall, are sacrificed during the oncologic surgery of advanced stage tumors. Previous reports have revealed that the amount of remnant oropharyngeal and palatal musculature after cancer resection decides the functional outcome of velopharyngeal competence [9]. Accurate measurement of the velopharyngeal orifice on pre- and postreconstructive state calculating size of the flap could improve the outcome. Further data from various patients should be collected. Procedures to achieve more natural velopharyngeal movement would be a matter of further study.

The large volume folded free flap and suspension with palmaris longus tendon for total soft palatal defects after oncologic surgery revealed a good prognosis for speech in the follow-up survey despite moderate hypernasality. More efforts should be made to restore natural velopharyngeal movement with dynamic reconstruction of the velopharyngeal structure. 


\section{REFERENCES}

1. Seikaly H, Rieger J, Wolfaardt J, et al. Functional outcomes after primary oropharyngeal cancer resection and reconstruction with the radial forearm free flap. Laryngoscope 2003;113:897-904.

2. Sinha UK, Young P, Hurvitz K, et al. Functional outcomes following palatal reconstruction with a folded radial forearm free flap. Ear Nose Throat J 2004;83:45-8.

3. Biglioli F, Brusati R. The folded radial forearm flap in softpalate and tonsillary fossa reconstruction: technical note. Int J Oral Maxillofac Surg 2008;37:76-81.

4. Brown JS, Zuydam AC, Jones DC, et al. Functional outcome in soft palate reconstruction using a radial forearm free flap in conjunction with a superiorly based pharyngeal flap. Head Neck 1997; 19:524-34.

5. McCombe D, Lyons B, Winkler R, et al. Speech and swallowing following radial forearm flap reconstruction of major soft palate defects. Br J Plast Surg 2005;58:306-11.

6. Roh TS, Lee WJ, Choi EC, et al. Radial forearm-palmaris longus tenocutaneous free flap; implication in the repair of the moderate-sized postoncologic soft palate defect. Head Neck 2009;31:1220-7.

7. Lew DH, Choi EC, Tark KC. Standardization of flap design for oropharyngeal reconstruction after cancer ablation surgery. Yonsei Med J 2003;44:1078-82.

8. Kim JH, Chu HR, Kang JM, et al. Functional benefit after modification of radial forearm free flap for soft palate reconstruction. Clin Exp Otorhinolaryngol 2008;1:161-5.

9. Rieger JM, Zalmanowitz JG, Li SY, et al. Speech outcomes after soft palate reconstruction with the soft palate insufficiency repair procedure. Head Neck 2008;30:1439-44.

10. Seikaly H, Rieger J, Zalmanowitz J, et al. Functional soft palate reconstruction: a comprehensive surgical approach. Head Neck 2008;30:1615-23. 